\title{
Studies on Stroke in Stroke-Prone Spontaneously Hypertensive Rat (SHRSP)
}

\section{(II) Cerebrovascular Fat Deposition and Cerebrovascular Permeability}

\author{
Yukio YAmori, ${ }^{*}$ Ryoichi Horie, ${ }^{* *}$ Kinya Otsubo, ${ }^{* *}$ Hajime HANDA, ${ }^{* *}$ \\ Masayasu Sato, ${ }^{* * *}$ and Kozo Окамото****
}

An extremely rapid development of ring-like fat deposition in the mesenteric arteries of spontaneously hypertensive rats (SHR) on high fat cholesterol diet was reported by Yamori (Tr Soc Path Jap 63:226, 1974) and further studied about its significance as a model for the studies on the initiation mechanism of atherosclerosis or arteriosclerosis by our previous reports (Jap Heart J 16:305, 1975). The process of ring-like fat deposition was speculated from some experimental findings as follows: Repetition of vasoconstriction and vasodilatation causes a segmental increase in vascular permeability, which is followed by ring-like fat deposition, especially in the media of vasodilated regions. The fat deposition accelerates the degeneration of arterial wall which further increases the permeability and results in the rapid development of marked ring-like lesions. In the present study, we examined such arterial fat depositions in the circle of Willis and basilar arteries in SHR and other experimental hypertensions and analyzed its relationship to extracerebral and intracerebral vascular permeability and some influential factors on the fat deposition.

\section{Material and Methods:}

(1) More than 200 rats of SHR, experimental hypertensive rats (EHR ; renal infarction and DOCA hypertension), and Wistar-Kyoto rats (WK) at the age of 60 days were fed on high fat cholesterol diet (HFCD; $20 \%$ of suet $5 \%$ of cholesterol and $2 \%$ of cholic acid) with or without $1 \%$ salt in drinking water for 1 to 10 weeks. Some group of SHR and EHR were treated with hydralazine hydrochloride $(80 \mathrm{mg} / \mathrm{L})$ in drinking water during hypercholesterolemic diet feeding.

(2) In order to observe the fat deposition in the circle of Willis " as a whole ", we utilized the following 3 methods: (I) microsurgical extirpation

From the Departments of Pathology, ${ }^{*}$ Neurosurgery, ${ }^{* *}$ and Psychiatry,*** Faculty of Medicine, Kyoto University, Kyoto; and Department of Pathology,**** Kinki University School of Medicine, Osaka-fu. 
of the circle of Willis and basilar arteries in SHR on HFCD, followed by Sudan III staining, (II) Sudan III staining of cerebral vessels from inside by perfusion with Sudan III solution following the preperfusion of $200 \mathrm{ml}$ of heparinized physiological saline $\left(\right.$ at $38^{\circ} \mathrm{C}$ ) with final systolic pressure of rats through an aortic cannula in SHR on HFCD, and (III) perfusion of $75 \%$ barium sulfate with $10 \%$ of gelatin after the above-mentioned physiological saline preperfusion, followed by Sudan III staining of the brains fixed with $10 \%$ formalin of SHR on HFCD and EHR on HFCD.

(3) To detect the relationship between increased permeability and fat deposition in SHR on HFCD, we injected $100 \mathrm{mg} / \mathrm{Kg}$ of horseradish peroxidase intravenously 2 hours prior to the intensive perfusion with saline through the aorta and the circle of Willis and basilar arteries were extirpated microsurgically for microscopical and electronmicroscopical observation on the portions with and without fat deposition after the diamino-benzidine reaction.

(4) The basilar artery was exposed in site by the microsurgical technique for vital observation on spasm in EHR and EHR on HFCD.

(5) For the qualitative detection of increased vascular permeability, SHR under pentobarbital anesthesia $(40 \mathrm{mg} / \mathrm{Kg}$, i.p.) were intensively perfused as mentioned above 2 to 4 hours after the intravenous infusion of (I) $3 \mathrm{ml} / \mathrm{Kg}$ of $0.5 \%$ trypanblue or (II) $40 \mathrm{mg} / \mathrm{Kg}$ of horseradish peroxidase in physiological saline. Removed brains were cut into several slices for the microscopical or vital microscopical observation on the leakage of the dye (I), or sectioned by a Vibratome into $50 \mu$ thick specimens which were stained by diamino-benzidine reaction for demonstration of the localization of peroxidase activity (II).

(6) Further, for the quantitative detection of increased permeability SHR were injected intravenously, (I) $30 \mu \mathrm{C} / \mathrm{Kg}$ of ${ }^{131} \mathrm{I}$ labelled human albumin (RISA) and (II) $40 \mathrm{mg} / \mathrm{Kg}$ of horseradish peroxidase, 24 hours prior to the intensive perfusion as described above. Brainstem and telencephalon (I), or the L-shaped cortical regions fed by anterior cerebral and posterior cerebral arteries, the other part of cortex fed by middle cerebral arteries and basal ganglia (II) were extirpated, weighed, homogenized for the extraction of radioactivity (I) (Yamori, Horie, Sato, Sasagawa, and Okamoto: Jap Circulat J 39:601, 1975) or of peroxidase, the activity of which was quantitatively assayed by spectrophotometrical determination of purpurogallin formation (II).

Results and Discussion:

(1) Such ring-like fat depositions as previously reported in the mesenteric arteries were clearly noted in the circle of Willis and basilar arteries " as 
a whole " in SHR on HFCD by the 3 afore-mentioned methods. These findings were apparently similar to a "ring-like" form of atherosclerosis in the circle of Willis and basilar arteries in human brains noted at autopsy.

(2) Regions with fat deposition in the circle of Willis showed the increased vascular permeability detectable by electron microscopic studies using peroxidase.

(3) Vasoconstriction seemed to be related to the ring-like fat deposition as the basic process causing ring-like increase in vascular permeability. Result (2) and (3) were discussed in detail in our previous reports (Jap Heart J $16: 305,1975)$

(4) The increased permeability of intracerebrovascular system was detected qualitatively as well as that of extracerebrovascular system (the circle of Willis) in SHR. The intracerebral localization of the increased permeability corresponded with the predilection sites of stroke in SHR. Such an increase could never be observed in WK.

(5) Further, quantitatively increased permeability of intracerebrovascular system was reasssured in SHRSP in the advanced stage of hypertension, especially, in the predilection sites of the areas fed by arteries with recurrent branching from the main stream as described previously (Jap Heart $\mathrm{J}$ $16: 329,1975)$.

(6) The number of sudanophilic rings in the circle of Willis was confirmed to show a linear statistical correlation to the grade of hypertension in SHR and EHR on HFCD.

(7) Ring-like fat depositions in the circle of Willis became marked and augmented by loading $1 \%$ salt in the drinking water in SHR on HFCD. When treated with hydralazine, no fat deposits were noted in EHR on HFGD while they were still detectable in SHR on HFCD. WK showed no fat depositions under any conditions.

\section{Summary:}

We devised the methods by which we could observe the ring-like fat deposition in the circle of Willis "as a whole" in SHR on HFCD.

This ring-like fat deposition is a good indicator for increased permeability in the vascular wall and this increased permeability seems to be related to vasospasm in cerebrovascular system in SHR. Further, we detected the increased permeability of intracerebrovascular system.

Our analysis of the factors affecting the fat deposition in the circle of Willis, obviously indicated the importance of hypertension and the acceleratory influence of increased salt intake. The possible involvement of some other factors affecting the arterial fat deposition still remained for the further 
study in SHR in contrast to EHR, in which hypertension itself seemed to be a main decisive factor for the fat deposition.

(This study was supported by Science and Technology Agency of Japanese Government, the Ministry of Education and Japan Society for the Promotion of Science.) 